\title{
Genetic relatedness among isolates of Shigella sonnei carrying class 2 integrons in Tehran, Iran, 2002-2003
} Reza Ranjbar ${ }^{\dagger 1}$, Aurora Aleo ${ }^{\dagger 2}$, Giovanni M Giammanco ${ }^{\dagger 2}$, Anna Maria Dionisi ${ }^{\dagger 3}$, Nourkhoda Sadeghifard ${ }^{\dagger 4}$ and Caterina Mammina*2

\begin{abstract}
Address: ${ }^{1}$ Research Center of Molecular Biology, Baqiyatallah University of Medical Sciences, Tehran, Iran, ${ }^{2}$ Dipartimento di Igiene e Microbiologia "G. D'Alessandro", Università di Palermo, Via del Vespro 133, I-90127 Palermo, Italy, ${ }^{3}$ Dipartimento di Malattie Infettive, Parassitarie e Immunomediate, Istituto Superiore di Sanità, Viale Regina Elena 299, I-00161Rome, Italy and ${ }^{4}$ Department of Microbiology, Faculty of Medicine, Ilam University of Medical Sciences, Ilam, Iran

Email: Reza Ranjbar - ranjbar@bmsu.ac.ir; Aurora Aleo - batteriologia@igiene.unipa.it; Giovanni M Giammanco - g.m.giammanco@unipa.it; Anna Maria Dionisi - annamaria.dionisi@iss.it; Nourkhoda Sadeghifard - sadeghifard@gmail.com; Caterina Mammina* - diptigmi@unipa.it

* Corresponding author †Equal contributors
\end{abstract}

Published: 22 June 2007

BMC Infectious Diseases 2007, 7:62 doi:10.1/86/1471-2334-7-62
Received: 20 February 2007

Accepted: 22 June 2007

This article is available from: http://www.biomedcentral.com/147I-2334/7/62

(C) 2007 Ranjbar et al; licensee BioMed Central Ltd.

This is an Open Access article distributed under the terms of the Creative Commons Attribution License (http://creativecommons.org/licenses/by/2.0), which permits unrestricted use, distribution, and reproduction in any medium, provided the original work is properly cited.

\begin{abstract}
Background: Shigella spp. are major cause of diarrhoeal disease in both developing and developed countries. Shigella sonnei is the serogroup of Shigella most frequently responsible for sporadic and epidemic enteritis in developed countries. In recent years the emergence and spread of S. sonnei biotype $g$ carrying class 2 integron have been frequently reported in many countries. Recently, $S$. sonnei has been reported as the prevalent serogroup of Shigella in Iran.

The present study was carried out to investigate phenotypic and genetic characteristics of Shigella sonnei isolates identified in the years 2002 and 2003 in Tehran, Iran.

Methods: Biotyping, drug susceptibility testing, pulsed field gel electrophoresis (PFGE) and analysis of class 2 integrons have been carried out on $60 \mathrm{~S}$. sonnei isolates, including 57 sporadic isolates from paediatric cases of shigellosis occurring in 2002 and 2003, two sporadic isolates recovered in 1984 and the ATCC 9290 strain.

Results: Biotype $g$ and resistance to streptomycin, sulfamethoxazole-trimethoprim and tetracycline were exhibited by 54 of the 57 recent isolates. Of the 54 biotype $g$ isolates, 28 exhibited a class 2 integron of 2161 bp, and 24 a class 2 integron of 1371 bp, respectively. Class 2 integrons were not detected in four isolates only, including the two endemic isolates recovered in 1984 and two strains from recent sporadic cases. PFGE divided the strains into eight pulsotypes labeled $\mathrm{A}$ to $\mathrm{H}$, three major pulsotypes - $\mathrm{A}$ to $\mathrm{C}$ - including the large majority of the recent sporadic $S$. sonnei isolates. Pulsotypes $A$ and $C$ were the most prevalent groups, accounting for $41.6 \%$ and $35.0 \%$, respectively, of the isolates under study.

Conclusion: The results suggest that biotype g, class 2 integron carrying $S$. sonnei are prevalent in our geographic area. S. sonnei isolated in the years 2002 and 2003 could be attributed to a few predominant clusters including, respectively, strains with pulsotypes $B$ and $C$ carrying a 2161 bp class 2 integron, and those having pulsotype $A$ and a $137 \mid$ bp class 2 integron. A few epidemic clones are responsible for the apparently endemic occurrence of shigellosis in Tehran, Iran.
\end{abstract}




\section{Background}

Infections caused by Shigella species are a major cause of diarrhoeal disease in both developing and developed countries. Globally, it is estimated that shigellosis causes about 1,100,000 deaths per year, two-thirds of the patients being children under 5 years of age $[1,2]$.

Development and spread of multidrug resistance is becoming a serious threat to Public Health worldwide since the early 1960s [3]. Mobile genetic elements, such as plasmids, transposons, genomic islands and integrons, disseminating via horizontal transfer are exponentially amplifying the problem [4]. In particular, the role of integrons in the dissemination of multidrug resistance in Gram-negative bacteria is well-established [5], and their carriage by several members of the family Enterobacteriaceae has been reported [6].

Recently, several reports have shown the successful emergence and spread in different countries of $S$. sonnei biotype g carrying a class 2 integron [4,7-12]. Pulsed Field Gel Electrophoresis (PFGE) analysis has proved that related groups of isolates may have circulating in recent years in different European countries [8]. Emergence and selective pressure by use of some antimicrobial agents or, alternatively, international trade are under debate as possible epidemiological mechanisms of the successful dissemination of this organism [13].

Shigellosis is one of the major causes of morbidity in children with diarrhea in Iran, but reports about prevalence of serogroups and molecular epidemiological features are limited. In two previous studies, S. flexneri has been reported as the most prevalent species in this country in the years 1984-'85 and 2001-'02 [14,15]. Similar patterns of serogroup prevalence have been detected in some neighboring countries. Indeed, S. flexneri, as the most frequent serogroup, accounted for $44.0 \%, 58.0 \%$, and $65.0 \%$, respectively, of shigellosis cases in Saudi Arabia [16], Pakistan [17] and Jordan [18]. In a subsequent investigation conducted in 2003, however, shifting of species distribution of Shigella appeared to occur in Tehran, Iran, where the proportion of $S$. sonnei isolates was 58.9 $\%$, while that of S. flexneri 36.4\% [19]. More recently, Farshad et al. (2006) has identified S. sonnei as the most prevalent Shigella species in Shiraz, Iran [20].

The present study was therefore undertaken to describe phenotypic and genetic characteristics of S. sonnei isolates identified in the years 2002-2003 in Tehran, Iran. Prevalence of biotypes, drug susceptibility patterns, pulsotypes and carriage of class 2 integrons was investigated with special attention to obtain an interpretative key of the apparently endemic occurrence of disease in this geographic area.

\section{Methods \\ Bacterial strains}

In the years 2002 and 2003, a total of 178 isolates of $S$. sonnei have been isolated from enteritis cases in children at five hospitals in Tehran, Iran. A random sample of 57 isolates was selected for the investigation. Three further strains were added: the S. sonnei strain ATCC 9290 and two apparently sporadic strains isolated in Tehran, Iran, in 1984.

All strains had been identified at a genus level by conventional methods by previously described procedures [21] while agglutination with specific antiserum from MAST Group LTD (Mast House, Derby Road, Bootle, Merseyside, L20 1EA, UK) was used to identify the species.

Biotyping was performed by the method of Nastasi et al. [21]. Antimicrobial susceptibility test was performed by the disk diffusion method according to the guidelines of the National Committee for Clinical Laboratory Standards [22]. The following antimicrobial agents were tested: ampicillin, AMP $(10 \mu \mathrm{g})$; ceftriaxone, CRO (30 $\mu \mathrm{g})$; kanamycin, K (30 $\mu \mathrm{g})$; streptomycin, STR (10 $\mu \mathrm{g})$; tetracycline, TET (30 $\mu \mathrm{g})$; ciprofloxacin, CP (5 $\mu \mathrm{g})$; nalidixic acid, NA $(30 \mu \mathrm{g})$; chloramphenicol, C $(30 \mu \mathrm{g})$; and sulfamethoxazole-trimethoprim, SXT (1.25/23.75 $\mu \mathrm{g})$. Escherichia coli ATCC 25922 was used as a quality control strain.

\section{Pulsed Field Gel Electrophoresis (PFGE) analysis}

All S. sonnei isolates were analyzed by PFGE with the restriction enzyme XbaI (Promega, Madison, WI, USA) under standardized conditions [23]. The Salmonella serotype Braenderup strain $\mathrm{H} 9812$ restricted with $\mathrm{XbaI}$ was used as molecular weight standard. Strain H9812 was kindly provided by the National Reference Centre for Enteric Pathogens at Istituto Superiore di Sanità, Rome, Italy.

PFGE patterns were initially visually assessed and interpreted by using the criteria established by Tenover et al. [24]. Computer-assisted analysis of the PFGE banding patterns was then performed with the Taxotron software (Taxolab, Institut Pasteur, Paris, France). Genetic distances were calculated using the Unweighted Pair Group Method using arithmetic Averages (UPGMA). Only bands whose molecular weight exceeded $90 \mathrm{Kbp}$ were considered and the calculated error was set at $10 \%$ for low molecular weight and progressively decreased to $5 \%$ for the highest weights $(1,000 \mathrm{Kbp})$.

\section{Class 2 integron analysis}

To detect the class 2 integrons, primer pair hep74 (5'CGGGATCCCGGACGGCATGCAC GATTTGTA-3') and hep51 (5'-GATGCCATCGCAAGTACGAG-3') was used under conditions described previously [8]. Amplified 
DNA products of interest were cloned by use of the TA cloning Kit (Invitrogen Life Technology, Italy) in invo $\mathrm{F}^{\prime}$ cells. The cloned products were sequenced by fluorescent dye-labeled dideoxynucleotides and a 373 automatic DNA sequencer (Perkin-Elmer, Foster City, CA), using the universal primers M13fw and M13rev for the first $1400 \mathrm{bp}$ and the primer ss1, 5'-AAGTGGCAGCAACGGATTCG-3', to complete the sequence. The resulting DNA sequence was analyzed by use of the BLAST search program 2.0 within the QBLAST system at the National Center for Biotechnology Information site.

\section{PFGE-Ceul and hybridization}

To establish if the class 2 integrons were located on the chromosome, the restriction enzyme CeuI was used to generate a pulsed-field gel electrophoresis (PFGE) profile to be hybridized with the int 2 sequence. $\mathrm{CeuI}$ is a rare-cutting enzyme, which produces very large restriction fragments. The preparation of genomic DNA of $S$. sonnei strains was performed according to the above protocol. Then, DNA restriction was done with $10 \mathrm{U} \mathrm{CeuI}$ enzyme (New England Biolabs, Beverly, MA, US) at $37^{\circ} \mathrm{C}$ for $3 \mathrm{~h}$ and DNA macrorestriction fragments were resolved on $0.7 \%$ agarose gel (Pulsed Field Certified, Bio-Rad, Hercules, CA, US) prepared in $0.5 \mathrm{X}$ TBE buffer $(50 \mathrm{mM}$ Tris, $50 \mathrm{mM}$ boric acid, $0.5 \mathrm{mM}$ EDTA). Lambda ladder concatamers (New England Biolabs) were used as molecular marker. The gel was run on a CHEF-DRII system (Bio-Rad Laboratories) under the conditions described by Liu et al. [25]. The gel, after staining for $10 \mathrm{~min}$ in ethidium bromide solution $(0.4 \mu \mathrm{g} / \mathrm{ml})$ and destaining in distilled water for $20 \mathrm{~min}$, was visualised under UV light and photographed.

Restricted fragments were transferred onto positively charged nylon membranes (Roche Diagnostics, Monza, Italy) by standard methods [25]. Southern blot hybridization was carried out under high-stringency conditions [26] using specific int 2 and 16sRNA probes, obtained amplifying the internal part of the respective genes with the following primers: int2, ssfw 5'-TTTCAGGTGGTGGGGAGATA-3' and ssrv 5'-TTGGTACAAAAGGCGTGACA-3'; 16sRNA, S16fw 5'-CAGCCACACTGGAACTGAGA-3' and S16rev 5'-GTTAGCCGGTGCTTCTTCTG-3'. Probes were labelled by the "PCR DIG labelling" kit (Roche Diagnostics).

\section{Results}

Fifty-four of the 57 isolates of $S$. sonnei identified in the period 2002-'03 and selected for the study were attributed to biotype $g$ and three only to biotype a. Both strains isolated in 1984 were biotype g (Additional file 1).

Additional file 1 summarizes the antimicrobial resistance phenotypes of the isolates under study. Fifty-four $S$. sonnei isolates obtained during the years 2002 and 2003 were resistant to streptomycin, sulfamethoxazole-trimethoprim and tetracycline. Additional resistances to nalidixic acid, ampicillin or kanamycin were carried by four, five and two isolates, respectively. Two further isolates showed ampicillin resistance associated to nalidixic acid or kanamycin, respectively. No isolates were resistant to ceftriaxone, ciprofloxacin, or chloramphenicol.

Three major clusters of $S$. sonnei were identified by XbaIPFGE among the sample of isolates recovered in Tehran, Iran, in 2002 and 2003 (Figure 1): PFGE type A ( $n=25$ isolates), PFGE type B ( $n=7$ isolates) and PFGE type C ( $n$ $=21$ isolates). Three subtypes $-\mathrm{a}$ to $\mathrm{c}-$ were included into PFGE type A, subtype a including 18 of the 25 strains. Two subtypes, $\mathrm{a}$ and $\mathrm{b}$, grouping five and two isolates, respectively, were observed among type B strains. Type C contained three subtypes, a to c, but subtype a included 15 of 21 isolates. The two isolates identified in 1984 were type E.

Two different class 2 integron structures were identified within the isolates under study. Twenty-eight (49.1\%) of the 57 isolates shared a 2161 bp class 2 integron, identified by DNA sequencing as containing four open reading frames, namely dhfrI, satI, aadA and orfX (99\% of homology with respect to the class 2 integron described by Pan JC et al, 2006). The second smaller integron of $1371 \mathrm{bp}$ containing only two genes within the cassettes, $d h f r I$ and satI, was detected in 24 strains (42.1\%). Alignments of the deduced amino acid sequences revealed two sequence substitutions in both gene cassettes, dhfrI and satI. The sequence of the class 2 integron of $1371 \mathrm{bp}$ has been submitted to GenBank (accession number AM745943).

Class 2 integrons were not detected in two strains from sporadic cases occurring in 2003 and in the two strains isolated in 1984. Probing using specific int 2 and 16sRNA probes confirmed the chromosomal location of both types of class 2 integrons (see additional file 2).

All isolates belonging to PFGE types B and C proved to possess the 2161 bp class 2 integron. Moreover, a closest association was found between pulsotype $A$ and the 1371 bp class 2 integron: indeed, all, but one, PFGE type A isolates were found to carry the smaller class 2 integron (Additional file 1 and Figure 1).

\section{Discussion}

Our study was undertaken to perform a baseline assessment of the epidemiologic features characterizing the endemic circulation of $S$. sonnei in Iran.

Biotype g included all, but three, recent isolates of $S$. sonnei. Emergence of biotype $\mathrm{g}$ or rhamnose negative strains 

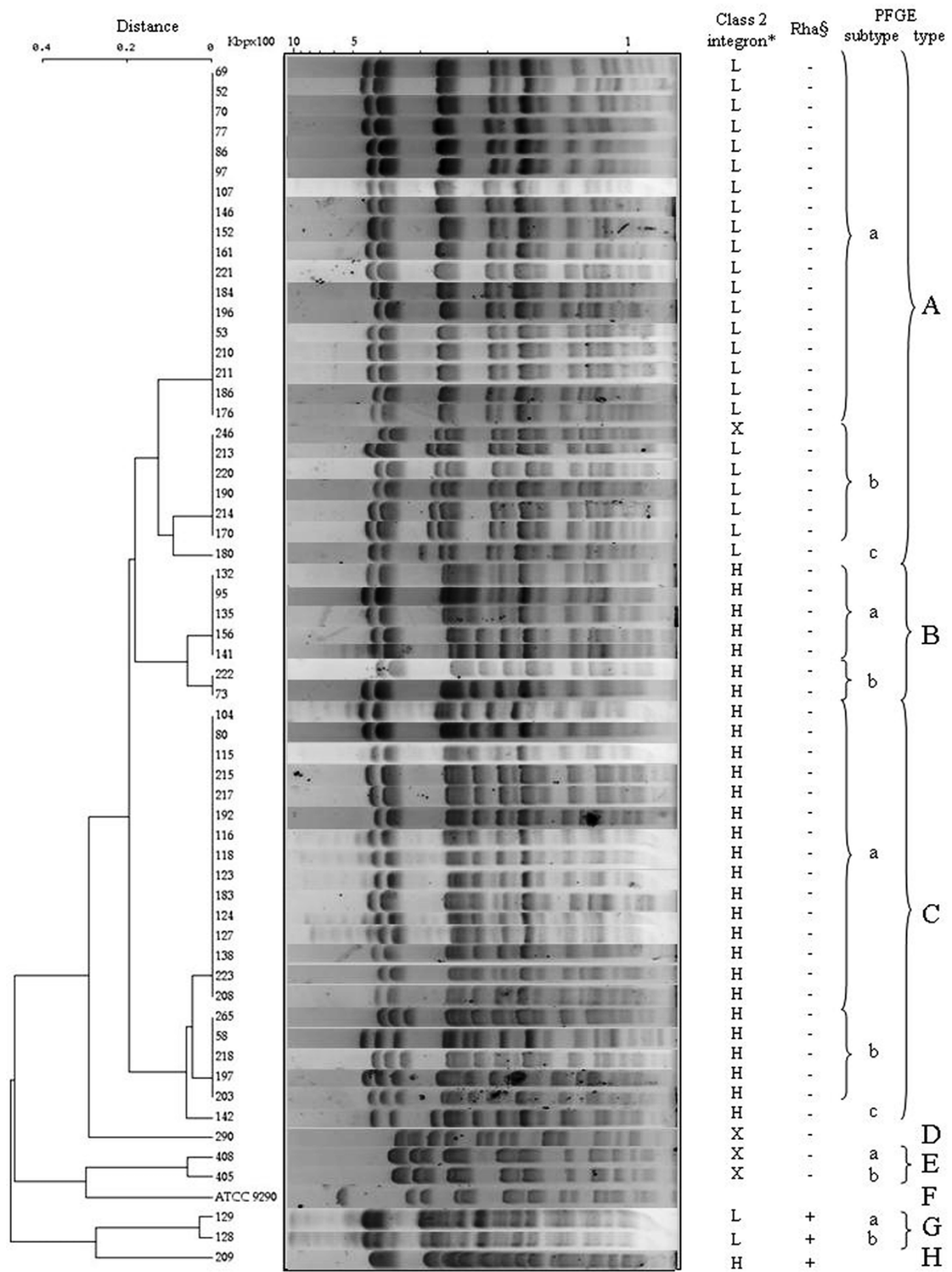

* $\mathrm{H}=$ high molecular weight class 2 integron; $\mathrm{L}=10 \mathrm{w}$ molecular weight class 2 integron; $\mathrm{X}=$ no class 2 integron. $\S$ Rhamnose fermentation.

Figure I

Phylogenetic dendrogram showing the genetic distances obtained from th comparison of the PFGE patterns produced using $X b a l$ restriction endonuclease from 59 Shigella sonnei isolates and from the type strain of the serogroup. 
has been repeatedly signaled in several countries from different continents in association with the characteristic resistance pattern streptomycin-trimethoprim frequently associated to tetracycline $[4,8,11]$. Moreover, antimicrobial susceptibility testing of the Iranian isolates showed a highest prevalence of a consistent resistance profile, accordingly to the reports from a number of countries $[3,6,28-31]$. These antibacterial drugs are commonly used worldwide in the treatment of dysentery. No resistance was observed to ciprofloxacin, ceftriaxone, and chloramphenicol, probably because of their very infrequent use as alternative therapeutic regimens in our geographic area. Selective pressure by indiscriminate use of antibiotics and mechanisms of horizontal gene transfer have undoubtedly contributed to the emergence and diffusion of multiresistance in Shigella [6].

Although other mechanisms are possible, resistance to streptomycin and trimethoprim seems to be attributable to expression of genes contained in class 2 integrons. These structures have proved to be highly prevalent within isolates of Shigella spp. from a number of countries $[4,6,8,10,11,14]$. Their gene array, generally including the open reading frames $d h f r I$, sat and $a a d$, is rather stable, because of the presence of a defective integrase, but some different structures have been very infrequently found in sporadic isolates of $S$. sonnei $[3,8,32]$. Our paper firstly describes the simultaneous circulation at approximately similar frequencies $(49.1 \%$ vs. $42.1 \%)$ in an apparently endemic context of two groups of strains carrying, respectively, a class 2 integron of $2161 \mathrm{bp}$ and a shorter integron, not containing the aad gene cassette sequence. No specific association was apparent with the resistance pattern.

When considering results obtained by PFGE, a first impressive feature is the limited genetic heterogeneity of the isolates under study, along with their clustering in a few predominant PFGE types. These findings could suggest that what is apparent as an endemic circulation of $S$. sonnei in Tehran, Iran, is more likely to be interpreted as an oligo-clonal epidemic. The paucity of types for a pathogen which has only humans as a significant reservoir of infection, might derive from few sources of infection in a context of epidemiological transition that, along with the recent switching of predominant serogroup from flexneri to sonnei, could suggest increasingly effective sanitary conditions in Tehran, Iran. On the other hand, success of $S$. sonnei is warranted also in developed countries by low infectious minimal dose, high secondary attack rates and, in comparison with the other serogroups, a better capability to survive to stressing environmental conditions and exploit alternative and more complex infectious routes, such as food vehicles [33-35].
When considering the association of phenotypic and genetic properties of multidrug resistance, our findings reinforce the previous considerations and suggest the involvement of our geographic area within an epidemiological global picture of emergence and/or dissemination of a limited number of well defined multiresistant clones of $S$. sonnei. The question whether a multicentric emergence under analogous selective factors of similar particularly "fit" organisms or, alternatively, a dissemination driven by the international trade of human and food related resources were the most scientifically sound mechanism, arises with special evidence, when considering the geographic scale of the phenomenon.

Molecular epidemiology provides a crucial contribution to accurately interpret epidemiological evolution of infectious diseases in communities, when screening by phenotypic methods, such as biotyping or drug resistance pattern analysis, is seriously hindered by the homogeneity of circulating strains.

\section{Conclusion}

The results suggest that biotype g, class 2 integron carrying $S$. sonnei are prevalent in our geographic area. S. sonnei isolated in the years 2002 and 2003 could be attributed to a few predominant clusters including, respectively, strains with pulsotypes $\mathrm{B}$ and $\mathrm{C}$ carrying a 2161 bp class 2 integron, and those having pulsotype $\mathrm{A}$ and a 1371 bp class 2 integron. A few epidemic clones are responsible for the apparently endemic occurrence of shigellosis in Tehran, Iran.

\section{Competing interests}

The author(s) declare that they have no competing interests.

\section{Authors' contributions}

RR and NS conceived the study, carried out biotyping, drug resistance analysis and drafted the manuscript. RR and AA performed PFGE analysis and detection of class 2 integrons. GMG carried out analysis of genetic similarity. AMD performed hybridization and sequencing experiments. CM participated in study design and coordination and helped to draft the manuscript. All authors read and approved the final manuscript.

\section{Additional material}

\section{Additional file 1}

Characteristics of S. sonnei isolates identified in Tehran, Iran, 20022003

Click here for file

[http://www.biomedcentral.com/content/supplementary/1471-

2334-7-62-S1.doc] 


\section{Additional file 2}

PFGE-CeuI and hybridization of representative isolates of $\mathrm{S}$. sonnei. DNA restriction was done with $10 U$ CeuI enzyme (New Englad Biolabs, Beverly, MA, US) at $37^{\circ} \mathrm{C}$ for $3 \mathrm{~h}$ and DNA macrorestriction fragments were resolved on $0.7 \%$ agarose gel (Pulsed Field Certified, Bio-Rad, Hercules, CA, US) suspended in 0.5X TBE buffer (50 $\mathrm{mM}$ Tris, $50 \mathrm{mM}$ boric acid, $0.5 \mathrm{mM}$ EDTA). Lambda ladder concatamers (New England Biolabs) were used as molecular marker. The gel was run on a CHEFDRII system (Bio-Rad Laboratories) under the conditions described by Liu et al. (1993). The gel was stained for $10 \mathrm{~min}$ in ethidium bromide solution $(0,4 \mu \mathrm{g} / \mathrm{ml})$, destained in distilled water for $20 \mathrm{~min}$, then visualised under UV light and photographed. Restricted fragments were transferred onto positively charged nylon membranes (Roche Diagnostics, Monza, Italy) by standard methods (Southern, 1975). Southern blot hybridization was carried out under high-stringency conditions, by using specific int 2 and 16sRNA probes obtained amplifying the internal portion of the respective genes and labelling the probes by the "PCR DIG labelling" kit (Roche Diagnostics).

Click here for file

[http://www.biomedcentral.com/content/supplementary/14712334-7-62-S2.ppt]

\section{Acknowledgements}

RR has been financially supported during his study period in Italy by the Research Center of Molecular Biology, Baqiyatallah University of Medical Sciences, Tehran, Iran

The authors would like to thank the staff of the Department of Hygiene and Microbiology, University of Palermo, for their helpful assistance in this study. The authors would also greatly appreciate the scientific contribute of Dr. Alessandra Carattoli.

\section{References}

I. Bennish ML, Wojtyniak BJ: Mortality due to shigellosis: community and hospital data. Rev Infect Dis 1991, I3(Suppl 4):S245-S25I.

2. Kotloff KL, Winickoff JP, Ivanoff B, Clemens JD, Swerdlow DL, Sansonetti PJ, Adak GK, Levine MM: Global burden of Shigella infections: implications for vaccine development and implementation of control strategies. Bull WHO 1999, 77:65I-666.

3. Ahmed AM, Furuta K, Shimomura K, Kasama $Y$, Shimamoto $T$ : Genetic characterization of multidrug resistance in Shigella spp. from Japan. J Med Microbiol 2006, 55:1685-169I.

4. Oh JY, Yu HS, Kim SK, Seol SY, Cho DT, Lee JC: Changes in patterns of antimicrobial susceptibility and integron carriage among Shigella sonnei isolates from southwestern Korea during epidemic periods. I Clin Microbiol 2003, 41 :42 I-423.

5. Hall RM, Stokes HW: Integrons: novel DNA elements which capture genes by site-specific recombination. Genetica 1993, 90:115-132.

6. Peirano G, Agers Ã Y, Aarestrup FM, dos Prazeres Rodrigues D: Occurrence of integrons and resistance genes among sulphonamide-resistant Shigella spp. from Brazil. J Antimicrob Chemother 2005, 55:30I-305.

7. Centers for Disease Control and Prevention (CDC): Day-care related outbreaks of rhamnose-negative Shigella sonnei - six states, June 200I-March 2003. MMWR 2004, 53:60-63.

8. DeLappe N, O'Halloran F, Fanning S, Corbett-Feeney G, Cheasty T, Cormican M: Antimicrobial resistance and genetic diversity of Shigella sonnei isolates from western Ireland, an area of low incidence of infection. J Clin Microbiol 2003, 41:1919-1924.

9. Hoe CH, Yasin RM, Koh YT, Thong KL: Antimicrobial susceptibility and pulsed-field gel electrophoresis of Shigella sonnei strains in Malaysia (1997-2000). J Appl Microbiol 2005, 99:133-140.

10. Mammina C, Pontello M, Dal Vecchio A, Nastasi A, Shigella sonnei Working Group: Identification of Shigella sonnei biotype g isolates carrying class 2 integrons in Italy (200 I to 2003). J Clin Microbiol 2005, 43:2467-2470.

II. Mclver CJ, White PA, Jones LA, Karagiannis T, Harkness J, Marriott D, Rawlinson WD: Epidemic strains of Shigella sonnei biotype $\mathbf{g}$ carrying integrons. J Clin Microbiol 2002, 40:1538-1540.

12. Terajima J, Tamura K, Hirose K, Izumiya H, Miyahara M, Konuma H, Watanabe H: A multi-prefectural outbreak of Shigella sonnei infections associated with eating oysters in Japan. Microbiol Immunol 2004, 48:49-52.

13. Memish ZA, Venkatesh S, Shibl AM: Impact of travel on international spread of antimicrobial resistance. Int J Antimicrob Agents 2003, 2 I: I35- I 42.

14. MoezArdalan K, Zali MR, Soltan- Dallal MM, Hemami MR, Salmanzadeh-Ahrabi S: Prevalence and pattern of antimicrobial resistance of Shigella species among patients with acute diarrhoea in Karaj, Tehran, Iran. J Health Popul Nutr 2003, $21: 96-102$.

15. Nikkah J, Mehr-Movahead A: Antibiotic resistance among Shigella species isolated in Tehran, Iran. Ann Trop Med Parasitol I988, 82:48I-483.

16. Kagalwalla AF, Khan SN, Kagalwalla YA, Alola S, Yaish HV: Childhood shigellosis in Saudi Arabia. Pediatr Infect Dis J 1992, II:215-219.

17. Zafar A, Sabir N, Bhutta ZA: Frequency of isolation of Shigella serogroups/serotypes and their antimicrobial susceptibility pattern in children from slum areas in Karachi. J Pak Med Assoc 2005, 55: I84- 188.

18. Rawashdeh MO, Ababneh AM, Shurman AA: Shigellosis in Jordanian children: A clinico-epidemiological prospective study and susceptibility to antibiotics. J Trop Pediatr 1994, 40:355-359.

19. Ranjbar R, Soltan Dallal MM, Pourshafie MR, Aslani MM, Majdzadeh R, Khorramizadeh MR: Serogroup distribution of Shigella spp. in Tehran. Iranian J Publ Health 2004, 33:32-35.

20. Farshad S, Sheikhi R, Japoni A, Basiri E, Alborzi A: Characterization of Shigella Strains in Iran by Plasmid Profile Analysis and PCR Amplification of ipa Genes. J Clin Microbiol 2006, 44:2879-2883.

21. Nastasi A, Pignato S, Mammina C, Giammanco G: rRNA gene restriction patterns and biotypes of Shigella sonnei. Epidemiol Infect 1993, I 1 0:23-30.

22. National Committee for Clinical Laboratory Standards: Performance standards for antimicrobial susceptibility testing. In Twelfth information supplement. Philadelphia: NCCLS; 2002:MI00-SI2.

23. Ribot EM, Fair MA, Gautom R, Cameron DN, Hunter SB, Swaminathan B, Barrett TJ: Standardization of pulsed-field gel electrophoresis protocols for the subtyping of Escherichia coli OI57:H7, Salmonella, and Shigella for PulseNet. Foodborne Pathog Dis 2006, 3:59-67.

24. Tenover FC, Arbeit RD, Goering RV, Mickelsen PA, Murray BE, Persing $\mathrm{DH}$, Swaminathan $\mathrm{B}$ : Interpreting chromosomal DNA restriction patterns produced by pulsed-field gel electrophoresis: criteria for bacterial strain typing. J Clin Microbiol 1995, 33:2233-2239.

25. Liu SL, Hessel A, Sanderson KE: Genomic mapping with I-Ceu I, an intron-encoded endonuclease specific for genes for ribosomal RNA, in Salmonella spp., Escherichia coli, and other bacteria. Proc Natl Acad Sci USA 1993, 90:6874-6878.

26. Southern EM: Detection of specific sequences among DNA fragments separated by electrophoresis. Mol Biol 1975, 98:503-5I7.

27. Pan JC, Ye R, Meng DM, Zhang W, Wang HQ, Liu KZ: Molecular characteristics of class $I$ and class 2 integrons and their relationships to antibiotic resistance in clinical isolates of Shigella sonnei and Shigella flexneri. J Antimicrob Chemother 2006, 58:288-96.

28. Dromigny JA, Macondo EA, Juergens-Behr A, Siby T, Perrier-GrosClaude JD: The distribution and antibiotic susceptibility of Shigella isolates in Dakar, Senegal (2000-2002). Int J Antimicrob Agents 2004, 24:307-308.

29. Fulla N, Prado V, Duran C, Lagos R, Levine MM: Surveillance for antimicrobial resistance profiles among Shigella species isolated from a semirural community in the northern adminis- 
trative area of Santiago, Chile. Am J Trop Med Hyg 2005, 72:85I-854.

30. Lima AA, Lima NL, Pinho MC, Barros Junior EA, Teixeira MJ, Martins $M C$, Guerrant RL: High frequency of strains multiply resistant to ampicillin, trimethoprim-sulfamethoxazole, streptomycin, chloramphenicol, and tetracycline isolated from patients with shigellosis in northeastern Brazil during the period 1988 to 1993. Antimicrob Agents Chemother 1995, 39:256-259.

31. Maraki S, Georgiladakis A, Christidou A, Scoulica E, Tselentis Y: Antimicrobial susceptibilities and b-lactamase production of Shigella isolates in Crete, Greece, during the period I9911995. APMIS 1998, 106:879-883.

32. Gassama-Sow A, Diallo MH, Boye CS, Garin B, Sire JM, Sow Al, Aidara-Kane $A$ : Class 2 integron-associated antibiotic resistance in Shigella sonnei isolates in Dakar, Senegal. Int J Antimicrob Agents 2006, 27:267-270.

33. Centers for Disease Control and Prevention (CDC): Outbreaks of Shigella sonnei infection associated with eating fresh parsley - United States and Canada, July-August 1998. MMWR Morb Mortal Wkly Rep 1999, 48:285-289.

34. Kapperud G, Rorvik LM, Hasseltvedt V, Høiby EA, Iversen BG, Staveland K, Johnsen G, Leitao J, Herikstad H, Andersson Y: Outbreak of Shigella sonnei infection traced to imported iceberg lettuce. J Clin Microbiol 1995, 33:609-6I4.

35. Nakamura M: The survival of Shigella sonnei on cotton, glass, wood, paper, and metal at various temperatures. J Hyg 1962, 60:35-39.

\section{Pre-publication history}

The pre-publication history for this paper can be accessed here:

http://www.biomedcentral.com/1471-2334/7/62/prepub

Publish with Biomed Central and every scientist can read your work free of charge

"BioMed Central will be the most significant development for disseminating the results of biomedical research in our lifetime. "

Sir Paul Nurse, Cancer Research UK

Your research papers will be:

- available free of charge to the entire biomedical community

- peer reviewed and published immediately upon acceptance

- cited in PubMed and archived on PubMed Central

- yours - you keep the copyright

Submit your manuscript here:

http://www.biomedcentral.com/info/publishing_adv.asp 